\title{
REGULARITY THEOREM FOR FUNCTIONS THAT ARE EXTREMAL TO PALEY INEQUALITY
}

\section{Seid Mohammed}

Department of Mathematics, Faculty of Science, Addis Ababa University PO Box 1176, Addis Ababa, Ethiopia, E-mail: maths.aau@telecom.net.et

ABSTRACT: In this paper we study the asymptotic behavior of functions that are extremal to the inequality introduced by Paley (1932) via a normal family of subharmonic functions.

Key words/phrases: Subharmonic, characteristic function, lower order, Pólya peaks, *-function.

\section{INTRODUCTION}

Let $\mathrm{u}$ be a subharmonc function in the complex plane. We set

$$
\begin{aligned}
& \mathrm{B}(\mathrm{r}, \mathrm{u})=\underset{|\mathrm{Z}|=r}{\sup u(z), \quad \mathrm{u}^{+}(\mathrm{z})=\max (\mathrm{u}(\mathrm{z}), 0) .} \\
& \mathrm{N}(\mathrm{r}, \mathrm{u})=\frac{1}{2 \pi} \int_{0}^{2 \pi} u\left(r e^{i^{0}}\right) d \theta
\end{aligned}
$$

and define the Nevanlinna characteristic of $u$ by $T(r)=T(r, u)=N\left(r, u^{+}\right)$.

The order $\rho$ of $\mathbf{u}$ is by definition

$$
\rho=\lim _{r \rightarrow \infty} \sup \frac{\log T(r, u)}{\log r}
$$

Paley's inequality asserts that

$$
\underline{\lim } \frac{B(r, u)}{T(r, u)} \leq \frac{\pi \rho}{\sin \pi \rho}
$$

provided $0<\rho \leq \frac{1}{2}$.

Inequality (1) was conjectured by Paley (1932) in the case where $u=\log |f(z)|$ for entire function $f$. Govorov (1969) proved Paley's conjecture. For a proof of (1) for general subharmonic $u$, see Essen (1975). The function 


$$
\mathrm{u}\left(\mathrm{re}^{\mathrm{i}^{\theta}}\right)=\frac{\pi \rho r^{\rho} \cos \rho \theta}{\sin \rho \pi},|\theta| \leq \pi
$$

in a subharmonic function which is extremal for (1) with

and

$$
T(r, u)=r^{p}
$$

$$
\mathrm{B}(\mathrm{r}, \mathrm{u})=\frac{\pi \rho r^{\rho}}{\sin \pi \rho}
$$

Indeed all subharmonic functions extremal for (1), i.e., for which equality holds in (1) behave asymptotically as (rotations of) the subharmonc function in (2). We shall prove,

Theorem 1: Let $u$ be a subharmonc function of order $\rho, 0<\rho \leq \frac{1}{2}$. If

$$
\varlimsup \frac{T(r, u)}{B(r, u)}=\frac{\sin \pi \rho}{\pi \rho}
$$

Then

$$
\begin{array}{ll}
\text { i) } & \mathrm{T}(\mathrm{r}, \mathrm{u})=\mathrm{r}^{\rho} \mathrm{L}(\mathrm{r}) \\
\text { ii) } & \mathrm{B}(\mathrm{r}, \mathrm{u}) \sim \frac{\pi \rho}{\sin \pi \rho} \mathrm{T}(\mathrm{r}, \mathrm{u}), \quad(\mathrm{r} \rightarrow \infty, \mathrm{r} \in \mathrm{G}) \\
\text { iii) } & \mathrm{N}(\mathrm{r}, \mathrm{u}) \sim \mathrm{T}(\mathrm{r}, \mathrm{u}), \quad(\mathrm{r} \rightarrow \infty, \mathrm{r} \in \mathrm{G}),
\end{array}
$$

where $L(r)$ varies slowly in a set $G$ of logarithmic density one, i.e.

$$
\lim _{\substack{r \in \infty \\ r \in G}} \frac{L(\sigma r)}{L(r)}=1, \quad(0<\sigma<\infty)
$$

holds (uniformly for $\sigma$ in any interval $A^{-1} \leq \sigma A, A>1$ ) with

$$
\mathrm{G}=\bigcup_{n=1}^{\infty}\left[a_{n}, b_{n}\right],\left(a_{n} \rightarrow \infty, \frac{b_{n}}{a_{n}} \rightarrow \infty\right)
$$

satisfying

$$
\int_{G \Gamma[1, r]} t^{-1} d t \sim \log \mathrm{r}, \quad(\mathrm{r} \rightarrow \infty) .
$$


Moreover, for any sequence $\left\{\mathrm{rn}_{\mathrm{n}} \subseteq \mathrm{G}\left(\mathrm{r}_{\mathrm{n}} \rightarrow \infty\right)\right.$ there is a subsequence of positive integers $\mathrm{I}=\{\mathrm{nk}\}$ and a real $\alpha \in[-\pi, \pi]$ such that for almost all $z$ in the set $\left\{\mathrm{re}^{\mathbf{i}^{\theta}},|\theta-\alpha|<\pi\right\}$ with respect to the Lebesgue measure of the plane we have

$$
\text { iv) } \quad \lim _{\substack{n \rightarrow \infty \\ n \in I}} \frac{u\left(r_{n} z\right)}{T\left(r_{n}, u\right)}=\frac{\pi \rho r^{\rho}}{\sin \pi \rho} \cos \rho(\theta-\alpha), \quad|\theta-\alpha| \leq \pi, \quad \mathrm{z}=\mathrm{re}^{\mathrm{i} \theta}
$$

\section{SOME FACTS}

Let $u$ be a subharmonic function in $\mathbb{C}$ of order $\rho, 0 \leq \rho<\infty$. By a sequence of Polya peaks for $T(r, u)$ of order $\rho$ we mean a sequence $\left\{r_{n}\right\}$ of positive numbers such that $\mathrm{T}(\mathrm{t}, \mathrm{u}) \leq\left(1+\varepsilon_{n}\right)\left(\frac{1}{r_{n}}\right)^{\rho} \mathrm{T}\left(\mathrm{r}_{n}, \mathrm{u}\right)$ holds for some sequence $\varepsilon_{n} \rightarrow 0, r \quad \varepsilon_{n} \rightarrow \infty$ and for all $t$, such that $\in_{n} r_{n} \leq t \leq r_{n} / \varepsilon_{n}$. If $\rho$ is finite $T(r, u)$ has a sequence of Polya peaks of order $\rho$ (for a proof see Edrei (1965)). We also need the *-function of $u$ introduced by Baernstein (1974) which is defined by

$$
\mathrm{u}^{*}\left(\mathrm{re}^{\mathrm{i} \theta}\right)=\frac{1}{2 \pi} \sup _{|E|=2 \theta} \int_{E} u\left(r e^{\Phi}\right) d \Phi,(0<\mathrm{r}<\infty, 0 \leq \infty \leq \pi),
$$

where $E \subseteq[-\pi, \pi]$ and $|E|=$ Lebesgue measure of the set $E$. It is shown that $u^{*}$ is subharmonic in the upper half plane, $\pi^{+}$and is continuous in the closure of $\pi^{+}$except possibly at the origin. It is also proved that if the function $\theta \rightarrow \tilde{u}\left(\mathrm{re}^{\mathrm{i}^{\theta}}\right)$, for fixed $\mathrm{r}>0$ and $|\theta| \leq \pi$, is the symmetric decreasing rearrangement of $u\left(r^{i \theta}\right)$, then

$$
\mathbf{u}^{\star}\left(\mathrm{ri}^{\mathbf{i}^{\theta}}\right)=\frac{1}{2 \pi} \int_{-\theta}^{\theta} \tilde{u}\left(\mathrm{re}^{i \phi}\right) \mathrm{d} \phi \quad(0 \leq \theta \leq \pi) .
$$

$u^{*}$ satisfies (see also Hayman (1989), chap. 9, especially p. 712).

and

$$
T(r, u)=\max _{0 \leq \theta \leq \pi} u^{*}\left(r e^{i \theta}\right), u^{*}(r)=0
$$

$$
\mathrm{B}(\mathrm{r}, \mathrm{u})=\left.\frac{\pi \partial u^{*}}{\partial \theta}\left(\mathrm{re}^{\mathrm{i}}\right)\right|_{\theta=0}=\tilde{u}(\mathrm{r})
$$

Proof of Theorem 1: First we shall establish assertions (i) and (ii) of Theorem 1. We use the well-known result due to Petrenko (1969) given below by Lemma 1 . 
Lemma 1. Suppose $u$ is subharmonic in $\mathbb{C}$. Fix $\gamma, 0<\gamma \leq 1$, and let

Then

$$
\mathrm{k}(\mathrm{t}, \gamma)=\frac{\gamma^{-2} t^{y_{r}}}{\left(t^{y_{r}}+1\right)^{2}}
$$

$$
\mathrm{B}(\mathrm{r}, \mathrm{u}) \leq \int_{0}^{R} u\left(t e^{i \pi \gamma}\right) \mathrm{k}(r / t, \gamma) \frac{d t}{t}+c\left(\frac{r}{R}\right)^{1 / t} \mathrm{~T}(2 \mathrm{R}, \mathrm{u}), \quad\left(0<\mathrm{r}<\frac{R}{2}\right),
$$

for an absolute constant $c$.

Besides Petrenko's (1969) proofs for Lemma 1 are given in Essen (1975) and in Edrei and Fuchs (1976, Lemma 11.1) where it was shown that

$$
\hat{k}(s, \gamma)=\int_{0}^{\infty} k(t, \gamma) \frac{d t}{t^{1+s}}=\frac{\pi s}{\sin (\pi \gamma s)},\left(0<r \frac{1}{\gamma}\right) \text {. }
$$

We apply Lemma 1 with $\gamma=1$ together with the hypotheses of Theorem 1 to obtain (on letting $R \rightarrow \infty$ ) in (7)

$$
\begin{aligned}
& \mathrm{T}(\mathrm{r}, \mathrm{u}) \leq\left(\frac{. \sin \pi \rho}{\pi \rho}+o(1)\right) \int_{0}^{\infty} \mathrm{T}(\mathrm{t}, \mathrm{u}) \mathrm{k}(\mathrm{r} / \mathrm{t}, \gamma) \frac{d t}{t},(\mathrm{r} \rightarrow \infty) \\
& =\left(\frac{\sin \pi \rho}{\pi \rho}+o(1)\right) \mathrm{T}(\mathrm{r}, \mathrm{u})^{*} \mathrm{k}(\mathrm{r}, \gamma) .
\end{aligned}
$$

Since $\hat{k}(\rho, \gamma)=\frac{\pi \rho}{\sin \pi \rho}$ and $\mathrm{T}(\mathrm{r}, \mathrm{u})$ is increasing it follows from the Drasin and Shea (1976) Tauberian theorem that there is a set $G$ of the from (4) such that

$$
T(r, u)=r^{\rho} L(r)
$$

where $L(r)$ varies slowly in $G$, and further

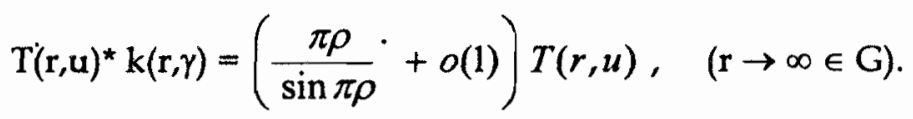

Thus from $(7),(10)$ and the hypotheses of Theorem 1 we conclude that

$$
\lim _{\substack{r \rightarrow \infty \\ r \in G}} \frac{B(r, u)}{T(r, u)}=\frac{\pi \rho}{\sin \pi \rho} \text {. }
$$


This proves assertions (i) and (ii) of Theorem 1. It also follows from (9) that any sequence $\left\{r_{n}\right\} \subseteq G, r_{n} \rightarrow \infty$, is a sequence of Polya peaks for $T(r, u)$ of order $\rho$ (see Edrei (1969) Lemma 4). We proceed to prove Theorem 1.

Let $\left\{r_{n}\right\}$ be any sequence of Polya peaks for $T(r, u)$ of order $\rho, 0 \leq \rho<\infty_{\text {. }}$ Then the sequence

$$
\mathrm{un}(\mathrm{z})=\frac{u\left(r_{n} z\right)}{T\left(r_{n} u\right)}(\mathrm{z} \in \mathbb{C}, \mathrm{n}=1,2,3, \ldots)
$$

forms a normal family of subharmonic functions in the sense of Anderson and Baernstein (1978), i.e., there is a subharmonic function $\mathrm{v}$ is $\mathbb{C}$ and a subsequence $I=\left\{n_{k}\right\}$ of positive integers such that
a) $\quad \lim _{\substack{n \rightarrow \infty \\ n \in 1}} \int_{0}^{2 \pi}\left|u_{n}\left(\mathrm{re}^{\mathrm{i}^{\theta}}\right)-\mathrm{v}\left(\mathrm{re}^{\mathrm{i}^{\theta}}\right)\right| \mathrm{d} \theta=0, \quad(0<\mathrm{r}<\infty)$
b) $\quad \lim _{\substack{n \rightarrow \infty \\ n \in l}} \mathrm{~T}\left(\mathrm{r}, \mathrm{u}_{\mathrm{n}}\right)=\lim _{\substack{n \rightarrow \infty \\ n \in l}} \frac{T\left(r r_{n}, u\right)}{T\left(r_{n}, u\right)}=\mathrm{T}(\mathrm{r}, \mathrm{v}) \leq \mathrm{r}^{\rho},(0<\mathrm{r}<\infty)$.

Thus if $u$ satisfies the hypotheses of Theorem 1 then from (9) and (13) we have

$$
\mathrm{T}(\mathrm{r}, \mathrm{v})=\mathrm{r}^{\mathrm{P}}
$$

Indeed we prove,

Theorem 2: Let $u$ be a subharmonic function that satisfies the hypotheses of Theorem 1 and $\left\{r_{n}\right\}$ a sequence of Polya peaks of $T(r, u)$ of order $p$, $0<\rho \leq \frac{1}{2}$. If $v$ is a subharmonic function that satisfies (13) corresponding to $u$ and the sequence $\left\{\mathrm{r}_{\mathrm{n}}\right\}$, then
a) $\quad T(r, v)=r^{\rho}=N(r, v)$
b) $\quad \mathrm{v}\left(\mathrm{re}^{\mathrm{i}^{\theta}}\right)=\frac{\pi \rho r^{\rho}}{\sin \pi \rho} \cos \rho(\theta-\alpha),|\theta-\alpha| \leq \pi$ for some $\alpha \in[-\pi, \pi]$.

It is clear that assertions (iii) and (iv) of Theorem 1 follow from Theorem 2 and (13). We first establish, 
Lemma 2. Suppose $u$ satisfies the hypotheses of Theorem 1 and $\left\{r_{n}\right\}$ is a sequence of Polya peaks of $T(r, u)$ of order $\rho, 0<\rho \leq \frac{1}{2}$. If $v$ is a subharmonic function that satisfies (13) corresponding to $\mathbf{u}$ and the sequence $\left\{r_{n}\right\}$ then $v^{\star}$ is harmonic in the upper half plane and

$$
v^{*}\left(\mathrm{re}^{\mathrm{i}^{\theta}}\right)=\mathrm{r}^{\rho} \frac{\sin \rho^{\theta}}{\sin \pi \rho} \quad(0 \leq \theta \leq \pi)
$$

Proof. First we prove $\mathrm{B}(\mathrm{r}, \mathrm{v})=\frac{\pi \rho r^{\rho}}{\sin \pi \rho}$. Since $\mathrm{v}^{*}\left(\mathrm{re}^{\left.\mathrm{i}^{\mathrm{i}}\right)}\right) \leq \mathrm{T}(\mathrm{r}, \mathrm{v})=\mathrm{r}^{\rho}$ and $\mathrm{v}^{*}(\mathrm{r})=0$ by (6) and (14), we have by Phragme'n - Lindeöf principle

$$
v^{*}\left(\mathrm{re}^{\mathrm{i}^{\theta}}\right) \leq \frac{r^{\rho} \sin \rho^{\theta} \theta}{\sin \pi \rho} \quad(0 \leq \theta \leq \pi)
$$

which implies $\left.\frac{\partial v^{*}}{\partial \theta}\left(\mathrm{re}^{\mathrm{i}^{\theta}}\right)\right|_{\theta=0} \leq \frac{\rho r^{\rho}}{\sin \pi \rho}$. Thus it follows from (6) that

$$
\mathrm{B}(\mathrm{r}, \mathrm{v}) \leq \frac{\pi \rho r^{\rho}}{\sin \pi \rho}
$$

To prove the reverse inequality we let $r>0$ and choose $\alpha_{n} \in[-\pi, \pi)$, $\left(n \in I=\left\{n_{k}\right\}\right)$ such that $B\left(r, u_{n}\right)=u_{n}\left(r e^{i^{\alpha}} n\right)$, where $u_{n}$ is defined by (12).

Assume $\alpha_{n} \rightarrow \alpha_{0}$ as $n \rightarrow \infty(n \in I)$. Then for any $s>r$, we have

$$
\mathrm{B}\left(\mathrm{r}, \mathrm{u}_{\mathrm{n}}\right) \leq \frac{1}{2 \pi} \int_{0}^{2 \pi} u_{n}\left(\mathrm{se}^{\mathrm{i}^{\theta}}\right) \mathrm{p}_{\mathrm{r} / \mathrm{s}}\left(\theta-\alpha_{n}\right) \mathrm{d} \theta
$$

where $\operatorname{\rho r}(\theta)$ is the Poisson kernel. By dominated convergence theorem and by (13) it follows (letting $n \rightarrow \infty, n \in I^{\prime}$ in (16)) that

$$
\frac{\pi \rho r^{\rho}}{\sin \pi \rho} \leq \frac{1}{2 \pi} \int_{0}^{2 \pi} v\left(s \mathrm{e}^{\mathrm{i} \theta}\right) \mathrm{p}_{\mathrm{r} / \mathrm{s}}\left(\theta-\alpha_{n}\right) \mathrm{d} \theta \leq \mathrm{B}(\mathrm{s}, v) .
$$

Since (17) holds for any $s>r$ and $B(s, v)$ is a continuous function of $s$, we have, letting $\mathrm{s} \rightarrow \mathrm{r}$ in $(17), \frac{\pi \rho r^{\rho}}{\sin \pi \rho} \leq \mathrm{B}(\mathrm{r}, v)$. Thus $\mathrm{B}(\mathrm{r}, \mathrm{v})=\frac{\pi \rho r^{\rho}}{\sin \pi \rho}$. To prove the lemma we apply (7) to $v$ with $\gamma=\frac{\alpha}{\pi}(0<\alpha<\pi)$, and using (16) we get 
$\frac{\pi \rho r^{\rho}}{\sin \pi \rho}=\mathrm{B}(\mathrm{r}, v) \leq \int_{0}^{\infty} v^{*}\left(\mathrm{te}^{\mathrm{i} \alpha}\right) \mathrm{k}(\mathrm{r} / \mathrm{t}, \gamma) \frac{d t^{4}}{t} \leq \frac{\sin \rho \alpha^{\infty}}{\sin \pi \rho} \int_{0}^{\rho} k\left(-r_{t}^{r}, \gamma\right) \frac{d t}{t}=\frac{\pi \rho r^{\rho}}{\sin \pi \rho}$

which implies $\mathrm{v}^{*}\left(\mathrm{te}^{\mathrm{i} \alpha}\right)=t^{\rho} \frac{\sin \rho \alpha}{\sin \pi \rho}$. Thus applying the maximum principle to (15) we conclude that $\mathrm{v}^{*}\left(\mathrm{re}^{\mathrm{i}^{\theta}}\right)=\frac{r^{\rho} \sin \rho^{\theta}}{\sin \pi \rho},(0 \leq \theta \leq \pi)$

To complete the proof of Theorem 2, let $\widetilde{v}$ be the symmetric decreasing rearrangement of $v$, so that

$$
v^{*}\left(\mathrm{re}^{\mathrm{i}^{\theta}}\right)=\frac{1}{2 \pi} \int_{-\theta}^{\theta} \widetilde{v}\left(\mathrm{re}^{\mathrm{i} \phi}\right), \quad(0 \leq \theta \leq \pi) .
$$

Since by Lemma $2 v^{*}(z)$ is harmonic in $\{z: 0<\arg (z)<\pi\}$, it follows from (18) that

$$
0=\Delta v^{*}\left(\mathrm{re}^{\mathrm{i}^{\theta}}\right)=\frac{1}{2 \pi} \int_{-\theta}^{\theta} \Delta \widetilde{v}\left(\mathrm{re}^{\mathrm{i} \phi}\right) \mathrm{d} \phi,(0<\theta<\pi)
$$

Thus $\widetilde{v}$ is harmonic in $\left\{\mathrm{re}^{i \theta}: \mathrm{r}>0,|\theta|<\pi\right]$. Let $\mathrm{r}_{1}>0$ and $\alpha \in[-\pi, \pi]$ be such that $\mathrm{v}\left(\mathrm{r}_{1} \mathrm{e}^{\mathrm{i}^{\alpha}}\right)=\mathrm{B}\left(\mathrm{r}_{1}, \mathrm{v}\right)=\tilde{\mathrm{k}}\left(\mathrm{r}_{1}\right)$. Then a comparison of $\mathrm{v}^{*}$ with the subharmonic function

$$
v_{\alpha}\left(\mathrm{re}^{\mathrm{i}^{\theta}}\right)=\frac{1}{2 \pi} \int_{-\theta+\alpha}^{\theta+\alpha} v\left(\mathrm{re}^{\mathrm{i} \phi}\right) \mathrm{d} \phi
$$

shows that (Essén and Shea, 1978/79)

$$
v\left(z \mathrm{e}^{\mathrm{j}^{\alpha}}\right)=\widetilde{v}(\mathrm{z}) .(|\arg (\mathrm{z})|<\pi) .
$$

By Lemma 2, (18) and (19) we get

$$
v\left(\mathrm{re}^{\mathrm{i} \theta}\right)=\frac{\pi \rho r^{\rho}}{\sin \pi \rho} \cos \rho(\theta-\alpha), \quad(|\theta-\alpha| \leq \pi) .
$$

\section{ACKNOWLEDGEMENTS}

I am very thankful for the reviewers whose suggestions upgraded the quality of the material and its presentation. 


\section{REFERENCES}

1. Anderson, J.M. and Baernstein, A. (1978). The size of the set of which'a meromorphic function is large II. Proc. London Math. Soc. 36:518-539.

2. Baernstein, A. (1974). Integral means, univalent functions and circular symmetrization II. Acta. Math. 133:139-169.

3. Drasinn, D. and Shea, D.F. (1976). Convolution inequalities, regular variations and exceptional sets. J. Analyse Math. 29:232-293.

4. Edrei, A. (1965). Sum of deficiencies of meromophic functions. J. Analyse Math. 14:79-107.

5. Edrei, A. (1969). Locally Tauberian theorems of mermorphic functions of lower order less than one, Trans. Amer. Math. Soc. 140 309-332.

6. Edrei, A. and Fuchs, W.H.J. (1976). Asymptotic behavior of mermorphic functions with extremal spread. Annales Academic Series A.I. Mathematica 2;67-111.

7. Essen, M. (1975). The cos $\pi \hat{\lambda}$-Theorem. Lect. Notes Math Springer, Berlin Heidelberg, $467 \mathrm{pp}$.

8. Essén, M. and Shea, D.F. (1978/79). On some questions of uniqueness in the Theory of Symmetrization, Ann. Acad. Sci. Fenn. Series 4:311-340.

9. Govorov, N.V. (1969). On Paley's problem ,in Russian. Funk. Anal. 3:35-40.

10. Hayman, W.K. (1989). Subharmonic Functions. Vol. 2, Academic Press, New York.

11. Paley, A. (1932). Note on integral function Proc. Cambridge Phil. Soc. 28:262-265.

12. Petrenko, V.P. (1969). The growth of meromophic functions of finite lower order. Izv. Akad. Navk. USSR 33:414-454. 